\title{
Simultaneous pancreas-kidney transplantation after liver transplantation: first Portuguese case report
}

Elsa Soares ${ }^{1, *}$, Rita Valério Alves ${ }^{2, *}$, Patrícia Cotovio ${ }^{3}$, Fernando Caeiro ${ }^{3}$, Inês Aires ${ }^{3}$, Miguel Bigotte ${ }^{3}$, Cecília Silva ${ }^{3}$, Ana Pena ${ }^{4}$, Luís Bicho $^{4}$, Américo Martins ${ }^{4}$, Francisco Remédio ${ }^{3}$, Aníbal Ferreira ${ }^{3}$, Fernando Nolasco ${ }^{3}$

* Equally contributing first authors

${ }^{1}$ Nephrology Department, Centro Hospitalar de Setúbal, Setubal.

${ }^{2}$ Nephrology Department, Centro Hospitalar Médio Tejo - Torres Novas.

${ }^{3}$ Nephrology Department, Centro Hospitalar Universitário de Lisboa Central, Lisboa;

${ }^{4}$ Surgery Department, Centro Hospitalar Universitário de Lisboa Central, Lisboa

\section{ABSTRACT}

Simultaneous kidney pancreas transplantation, if feasible, is the treatment of choice for the management of end-stage renal disease in patients with type 1 diabetes. This intervention can offer glycemic control and might also stabilize or even reverse some of the metabolic complications of diabetes.

Chronic kidney disease has become a critical problem in orthotopic liver transplantation due to multiple risk factors, including post-transplant diabetes mellitus and immunosuppression. This has a major impact on graft and patient survival.

In this paper we present the case of a 42 -year-old man submitted to a simultaneous pancreas kidney transplant after a successful liver transplantation 18 years earlier. The surgical procedure was uneventful, and the patient was discharged 18 days after admission. The case findings support that simultaneous pancreas and kidney transplantation is an effective method for the treatment of chronic kidney disease in patients with diabetes and a previous liver transplant.

Keywords: Chronic kidney disease; Diabetes mellitus type 1; Orthotopic liver transplantation, Simultaneous pancreas kidney transplantation

\section{INTRODUCTION}

Kidney transplantation is the treatment of choice for the management of end-stage renal disease (ESRD). ${ }^{1}$ However, in patients with diabetes mellitus (DM) the underlying metabolic disturbance will persist and may even worsen after isolated kidney transplantation. ${ }^{1}$

The treatment of patients with DM presents many challenges to care providers. ${ }^{2}$ Pancreatic transplantation in humans was first introduced in 1966 and the initial outcome was disappointing. ${ }^{1}$ However, pancreas transplantation has undergone a number of important surgical and medical advances leading to improved outcomes, particularly in recent years. ${ }^{1,3}$ The most common type of pancreas transplant is simultaneous pancreas kidney (SPK) transplantation. The procedure is usually performed in patients with insulin-treated DM, an estimated glomerular filtration rate lower than $20 \mathrm{~mL} / \mathrm{min} / 1.73 \mathrm{~m}^{2}$ and good cardiovascular fitness. ${ }^{2,4}$

There are no large randomized controlled studies comparing the outcomes of SPK to kidney transplant alone (KTA). Registry data analysis are therefore the basis for most comparisons, which can be limited by patient selection bias. ${ }^{5}$ The most stringent retrospective comparisons support SPK transplantation over KTA for patients with DM and chronic kidney disease (CKD), ${ }^{1,3}$ as SPK transplantation might not only solve the problem of organ failure, but also stabilize or even reverse the metabolic complications of DM. ${ }^{1,5}$

Recent studies in pancreas transplantation provided evidence that the glycemic control associated with pancreas transplantation leads to improvement in endothelial function, reduction in nephropathic microvascular changes, and a reduction in cardiovascular mortality. ${ }^{3}$

Liver transplant recipients with stage 5 CKD and insulin-dependent DM, on high dose insulin therapy, might be suitable candidates for SPK transplantation. ${ }^{6}$ The selection criteria for this procedure are similar to those adopted in SPK candidates without liver transplant. However, as this procedure is technically demanding and experience is still limited, it is usually required that patients have a relative young age and high surgical tolerability to be considered for this intervention. ${ }^{6}$

\section{CASE REPORT}

We present the case of a 42-year-old leucodermic male patient, with type $1 \mathrm{DM}$ diagnosed at age 18 with a past history of poor metabolic control and diabetic nephropathy. Other medical conditions included arterial hypertension, status post-cholecystectomy, status 
post-Roux-en-Y hepaticojejunostomy and status post-orthotopic liver transplantation (OLT).

He had been submitted to urgent cholecystectomy at age 7 but the cause had not been completely established. Three years later, at age 10 , he presented with abdominal dilation, jaundice and ascites and was then submitted to Roux-en-Y hepaticojejunostomy. At 24-years-old, the patient underwent OLT surgery at Curry Cabral Hospital due to serious liver failure resulting from secondary biliary cirrhosis due to the previous surgeries. At 35-years-old, the patient was diagnosed with CKD and kidney biopsy showed diabetic nephropathy and calcineurin inhibitor (CNIs) toxicity. Liver graft function was unremarkable.

The patient was evaluated at Curry Cabral Hospital for pre-emptive SPK transplantation in early 2019. Investigations on secondary causes of insulin-dependent DM showed undetectable $C$ peptide levels and positive anti-glutamic acid decarboxylase antibodies, consistent with type $1 \mathrm{DM}^{7}$. After evaluation by the surgical team and overlooking the surgical complexity, abdominal computed tomography and magnetic resonance imaging scans were performed. The patient was included on the waiting list in October 2019, at that time with a serum creatinine $(\mathrm{pCr})$ of $3.3 \mathrm{mg} / \mathrm{dL}$, serum urea $(\mathrm{pUr})$ of $243 \mathrm{mg} / \mathrm{dL}$ and eGFR (CKD-EPI formula) $22 \mathrm{ml} / \mathrm{min} / 1.73 \mathrm{~m}^{2}$.

Although a longer waiting period was expected, given that he was blood type $\mathrm{O}$ and isoimmunised (panel-reactive antibody (PRA) of 24 $\%$ due to prior transfusions and transplantation), two months after joining the list he was summoned for SPK transplantation.

At admission, his medications consisted of mycophenolic acid (MPA) $360 \mathrm{mg}$ twice a day (BID), prednisolone $15 \mathrm{mg}$ once daily (OD), carvedilol $25 \mathrm{mg}$ (BID), perindopril $5 \mathrm{mg}(\mathrm{OD})$, clonidine $0.15 \mathrm{mg}$ (BID), furosemide $80 \mathrm{mg}$ (BID); allopurinol $100 \mathrm{mg}$ (OD), esomeprazole $40 \mathrm{mg}$ (OD); epoetin beta 10000 units per week and isophane insulin (38 IU OD).

The donor was a 49-year-old female who suffered an ischemic stroke. The donor-recipient pair showed 3 human leucocyte antigen (HLA) A, B, DR mismatches (respectively,1-1-1) and the initial crossmatch was negative. Donor and recipient were both cytomegalovirus (CMV) antibody positive.

The induction immunosuppression protocol consisted of thymoglobulin (cumulative dose $9.9 \mathrm{mg} / \mathrm{kg}$ ), MPA $180 \mathrm{mg}$ (BID), three methylprednisolone pulses $500 \mathrm{mg} /$ day followed by prednisolone $20 \mathrm{mg}$ (OD). Maintenance immunosuppression included prednisolone, MPA and tacrolimus. The patient also received the following prophylaxis: valganciclovir $450 \mathrm{mg}(\mathrm{OD})$, trimethoprim-sulfamethoxazole $480 \mathrm{mg}(\mathrm{OD})$, enoxaparin $500 \mathrm{UI} / \mathrm{h}$ and ceftazidime $1 \mathrm{~g} /$ day according to unit protocol.

Surgery was uneventful and he had immediate diuresis. On the $1^{\text {st }}$ post-operative day, he presented hemodynamic instability requiring vasopressors and transfusion support ( 2 red blood cell units). Immediate graft ultrasound was performed and a massive perirenal hematoma was detected. The patient was then submitted to surgical reintervention for drainage, after which he remained hemodynamically stable. On the $6^{\text {th }}$ post-operative day, he was transferred from the intensive care unit to the transplantation ward.
Regarding the renal graft, there was a rapid decrease in $\mathrm{pCr}$ with a nadir of $0.89 \mathrm{mg} / \mathrm{dL}$ at day six, and serial Doppler ultrasound showed normal graft perfusion. Pancreatic graft function recover was slower. Although serum lipase, amylase and $C$ peptide levels were progressively normalised, insulin therapy was needed to achieve glycemic control. Pancreatic Doppler ultrasound was requested but bowel interposition did not allow this assessment.

Immunological testing for donor-specific antibodies (DSAsLUMINEX) and CDC crossmatch were performed pre-transplant, after 48 hours and at day 8 post-transplant. They all showed negative results. Donor-specific anti-HLA antibodies were searched for, based on a single antigen bead assay (Luminex), that permits detection of a great number of anti-HLA antibodies in one test. ${ }^{8}$

Liver graft function was never affected (no changes were recorded on coagulation tests or liver enzyme levels).

At $6^{\text {th }}$ day he presented with fever and dyspnea, and analytically with leukocytosis and increased C-Reactive Protein (78 mg/L). Investigation was consistent with a nosocomial pneumonia, although no infectious agent was identified. He was treated empirically with vancomycin during 10 days with levels maintained between 10 and 15 $\mu \mathrm{g} / \mathrm{mL}$ and piperacillin/tazobactam (4.500g every 8-hours for 7 days) with complete resolution of symptoms and laboratory test normalization.

Eighteen days after SPK transplantation he was discharged to the outpatient clinic with good renal graft function $(\mathrm{pCr}$ of $0.82 \mathrm{mg} / \mathrm{dL}$, pUr of $61 \mathrm{mg} / \mathrm{dL}$; eGFR (CKD-EPI formula) $109 \mathrm{~mL} / \mathrm{min} / 1.73 \mathrm{~m}^{2}$ ) but still requiring insulin therapy (24 IU per day).

At follow-up, 18 years after liver transplantation and 4 months after SPK transplantation, liver and kidney grafts showed excellent function with normal laboratory parameters. Pancreatic graft function improved with reduced need for insulin therapy (7 IU/ OD), and his glycosylated hemoglobin was $6.7 \%$ with C peptide of $2.1 \mathrm{ng} / \mathrm{mL}$.

At this point immunosuppression was MPA $360 \mathrm{mg}$ (BID), prednisolone $5 \mathrm{mg}(\mathrm{OD})$ and tacrolimus $0.5 \mathrm{mg}$ (BID).

\section{DISCUSSION}

To the best of our knowledge, this is the first case of SPK transplantation for the treatment of type 1 DM and CKD after liver transplantation, in Portugal. Three similar case reports were found in literature, from Austria and China. 5,6

In non-renal solid organ transplants, CKD has become a critical problem due to multiple risk factors, including DM and immunosuppression. ${ }^{6} \mathrm{CKD}$ is a common complication and has a major impact on graft and patient survival. ${ }^{5-9}$ Although immunosuppressive therapy with CNIs has dramatically extended patient survival after OLT, CKD due to $\mathrm{CNI}$-associated nephrotoxicity is a common adverse event affecting morbidity as well as mortality. ${ }^{9}$ Regarding our patient, both $\mathrm{DM}$ and $\mathrm{CNI}$-associated nephrotoxicity were pathogenic factors for the development of CKD, as shown in the kidney biopsy. Probably, the 
poor metabolic control and the development of diabetic nephropathy were also exacerbated by the diabetogenic effects of immunosuppression after OLT.

Liver transplant recipients with CKD stage 5 and insulin-dependent DM might be suitable candidates for SPK transplantation. Nevertheless, the immunological challenge is huge as such patients may receive different grafts from different donors. ${ }^{6}$ As described in this case report, this patient had a previous history of blood transfusions and an elevated PRA (24\%), which could jeopardize transplantation results. So far, the chosen immunosuppression regimen has proven efficacy and safe as no rejection episodes or serious adverse effects have been reported up to this point.

There are case reports of en bloc transplantation of the liver, pancreas and both kidneys for the management of life-threating complications of the rare genetic disease Wolcott-Rallison Syndrome. En bloc transplantation is a technically challenging procedure where all organs are transplanted together while connected to the same-shared vasculature. ${ }^{10}$ With adequate technical and surgical expertise, the procedure should be considered a first-line treatment of choice for patients with multiple organ failure. ${ }^{11,12}$

This transplantation technique may have some advantages over separate implantation of both organs in patients with type $1 \mathrm{DM}$ and end-stage liver disease, including better long-term survival and better quality of life after transplantation. ${ }^{13}$ Moreover, combined transplant can prevent more serious rejection episodes due to the immunotolerant effect of a liver allograft. ${ }^{13}$ However, our patient had no signs of CKD at the time of liver transplantation and neither a multivisceral nor simultaneous liver-kidney transplantation was indicated at that time.

We consider this case deserves to be highlighted since, to the best of our knowledge, it is the first SPK transplantation performed in a patient with previous OLT in Portugal. Note that SPK transplantation, which is technically safe and feasible, can enhance the long-term outcome after liver transplantation and presents the advantage of simultaneously treating two major risk factors for post-OLT survival: DM and CKD.

Disclosure of potential conflicts of interest: none declared

\section{References}

1. Aref A, Zayan T, Pararajasingam R, Sharma A, Halawa A. Pancreatic transplantation: Brief review of the current evidence. World J Transplant. 2019;9(4):81-93.

2. Dean PG, Kukla A, Stegall MD, Kudva YC. Pancreas transplantation. BMJ. 2017;357:1-11.

3. Stites E, Kennealey P, Wiseman AC. Current status of pancreas transplantation. Curr Opin Nephrol Hypertens. 2016;25(6):563-569.

4. Flatt AJS, Bennett D, Counter C, Brown AL, White SA, Shaw JAM. $\beta$-Cell and renal transplantation options for diabetes. Diabet Med. 2020;37(4):580-592.

5. Stites E, Wiseman AC. Multiorgan transplantation. Transplant Rev [Internet]. 2016;30(4):253-60. Available from: http://dx.doi.org/10.1016/j.trre.2016.04.002

6. Tam N, Zhang C, Lin J, Wu C, Deng R, Liao B, et al. Simultaneous pancreas and kidney transplantation for liver transplant recipients with diabetes and uremia. Clin Res Hepatol Gastroenterol [Internet]. 2015;39(3):399-404. Available from: http://dx.doi.org/10.1016/j.clinre.2014.10.001

7. Leighton E, Sainsbury CA, Jones GC. A practical review of C-peptide testing in diabetes. Diabetes Ther. 2017:8(3):475-487.

8. McCaughan J, Xu Q, Tinckam K. Detecting donor-specific antibodies: the importance of sorting the wheat from the chaff. HepatoBiliary Surg Nutr. 2019;8(1):37-52.

9. Marschalek J, Györi GP, Silberhumer GR, Jomrich G, Kristo I, Steininger R, et al. Simultaneous pancreas-kidney transplantation nine years after liver transplantation - A case report. Transplant Proc [Internet]. 2012;44(10):3041-3043. Available from: http://dx.doi.org/10.1016/j.transproceed.2012.06.068

10. Tzakis AG, Nunnelley MJ, Tekin A, Buccini LD, Garcia J, Uchida K, et al. Liver, pancreas and kidney transplantation for the treatment of Wolcott-Rallison syndrome. Am J Transplant. 2015;15(2):565567.

11. Mieles L, Todo S, Tzakis A, TES. Treatment of upper abdominal malignancies with organ cluster procedures. Clin Transplant. 1990;4(2):63-67.

12. Caicedo LA, Villegas JI, Serrano O, Millán M, Sepúlveda M, Jiménez D, et al. En-bloc transplant of the liver, kidney and pancreas: experience from a Latin American transplant center. Am J Case Rep. 2017;18:114-118.

13. Nikeghbalian S, Mehdi SH, Aliakbarian M, Kazemi K, Shamsaeefar A, Bahreini A, et al. En-bloc liver-pancreas transplant in Iran. Arch Iran Med. 2014;17(9):640-641.

\section{Correspondence to:}

Elsa Soares, MD

E-mail: elsa.qsoares@gmail.com

Rita Valério Alves, MD

E-mail: anarita1990@gmail.com 